\title{
Time domain electromagnetic migration imaging
}

\author{
Shuming Wang \\ Institute of geophysics \& geomatics, China university of \\ geoscience \\ Wuhan, China \\ whwsm@hotmail.com \\ Qingyun Di \\ Institute of geology and geophysics, Chinese academy of \\ sciences \\ Beijing, China \\ qydi@mail.iggcas.ac.cn
}

\author{
Wenxiu Yuan \\ Institute of geophysics \& geomatics, China university of \\ geoscience \\ Wuhan, China \\ 1127360835@qq.com \\ Ruo Wang \\ Institute of geology and geophysics, Chinese academy of \\ sciences \\ Beijing, China \\ wangruo@mail.iggcas.ac.cn
}

\begin{abstract}
This article is about the study of time domain electromagnetic data migration imaging. First, based on the electromagnetic diffusion theory and the boundary conditions, we deduce the analytical formula of electromagnetic migration field. Then, we complete the calculation of electromagnetic migration field filter by electromagnetic migration field theory in frequency domain. The analytical electromagnetic migration filter is discretized to achieve electromagnetic numerical migration filter to make it able to be applied into practical discrete sampling. By analyzing the error caused by of discretization procedure according to the theory of electromagnetic migration filter, we deduce discretization error correction formula. Moreover, we study and realize the transform from time domain to frequency domain, and make it able to be used in time domain electromagnetic migration imaging. In order to test migration imaging technology, whole space $2 \mathrm{D}$ or $3 \mathrm{D}$ geoelectric models are built with the transmiter-receiver distance varying from near field to several thousand kilometers. Using the technology presented by this paper to the numeric data, the reasonable position of underground exploration body can be recovered. Finally, we deduce 2D migration field analytical formula in time domain and discretize it, then complete the numeric calculation in time domain directly. We analyze the kernel function of time domain electromagnetic migration filter and its influence factors, and test the effect of time domain migration field imaging by several simple geoelectric models.
\end{abstract}

Keywords - time domain; electromagnetic; migration; $2 D ; 3 D$

\section{INTRODUCTION}

The study on migration imaging of electromagnetic data based on diffusion equation was originated abroad. It has been experienced nearly three decades since Zhdanov ${ }^{[1,2]}$ and others proposed the theory of migration imaging of electromagnetic data. The theory becomes more and more mature, and the technique becomes more abundant. The migration field can be calculated using different methods such as integral transform and finite difference, also resistivity imaging can be achieved using different ways as the condition of reflection function and inversion. In terms of the complexity of geology structure, 2D and 3D migration imaging are realized reasonably in frequency domain $^{[3]}$. Direct time domain migration imaging is researched mainly on two-dimensional structures, however the theory still can be extended to the cases of 2.5-dimensional and 3dimensional. In practice, the method has been applied to process practical data abroad, and they have gotten some reasonable results. This Confirmed that the technology has potential to be applied in the practical electromagnetic data processing and interpretation ${ }^{[4,5]}$. Time-domain electromagnetic migration imaging is scarcely studied in China. Guoyin Lv studied the time-domain electromagnetic migration without the migration imaging, and only proposed determining the optimal time of migration layer by layer based the theory of electromagnetic migration proposed by Zhdanov ${ }^{[6]}$.

In this paper, we derived the analytic expression of electromagnetic migration field combined with the boundary conditions based on the theory of electromagnetic diffusion, and realized the theoretical filter by calculating electromagnetic migration field and discretized the theoretical filter ${ }^{[6}$. Then the error generated during the discretization was analyzed, and the selection principle of optimum filter parameter was determined. In order to test the filter functions, firstly we built different 2D and $3 \mathrm{D}$ geoelectric models in whole space with the separation between receiver and transmitter from near field until thousands of kilometers, and performed numerical calculation to gain simulative data[7]. Then we processed the data using the technique of electromagnetic migration and provide really reasonable information about the position of subsurface exploration target ${ }^{[8]}$.

\section{Theory of Time Domain Electromagnetic Migration}

Time Domain Electromagnetic migration is studied in time domain, and its principle is based on the electromagnetic migration in reverse time ${ }^{[9]}$. According to the theory of electromagnetic migration, electromagnetic migration fields satisfy the following formula: 


$$
\left\{\begin{array}{l}
P^{m}(\vec{r}, \tau)=P^{0}(\vec{r}, T-\tau) \\
\nabla^{2} P^{m}(\vec{r}, \tau)-\mu_{0} \sigma_{b} \frac{\partial P^{m}(\vec{r}, \tau)}{\partial \tau}=0, z>0 \\
P^{m}(\vec{r}, \tau) \rightarrow 0, \text { 当 } \vec{r} \rightarrow \infty, z>0
\end{array}\right.
$$

If the time parameter in formula is replaced by the migration time $t$, then the formula will have the following form.

$$
\nabla^{2} P^{\mathrm{m}}(\overrightarrow{\mathrm{r}}, T-t)+\mu_{0} \sigma_{b} \frac{\partial P^{\mathrm{m}}(\overrightarrow{\mathrm{r}}, T-t)}{\partial t}=0, z>0
$$

The above formula can be considered as the adjoint equation of diffusion equation, and describe the process of migration field propagating from the receiving position to field source ${ }^{[10]}$. So the problem of migration field calculation turns to the process of continuation of electromagnetic field observed on the ground in reverse time. In other words, the time domain electromagnetic migration field calculation turns to solve the equations which satisfy above boundary value.

\section{A. Frequency Domain Migration Imaging Numerical Experiment}

By the field transformation, we can use simulation in the frequency domain to achieve time domain numerical simulation. Similarly, the frequency domain interpretation method can be used on the interpretation of time domain data. One kind of time domain electromagnetic migration method is based on the field transformation. Firstly, reading time-domain electromagnetic observational data, then transforming time domain electromagnetic signal to the frequency domain. After that, the migration filter can be used to calculate the corresponding value of migration field in frequency domain. Finding the background field, and calculating the reflection

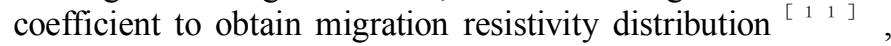
then we use numerical methods to test the method used in this study. The condition is based on the reflex function of migration imaging as follows:

$$
\beta_{\mathrm{x}, y}\left(\mathrm{x}, \mathrm{y}, \mathrm{z}, \omega_{l}\right)=\frac{E_{x, y}^{m}\left(x, y, z, \varpi_{l}\right)}{E_{x, y}^{\mathrm{d}}(x, y, z, \varpi)}, l=1,2, \cdots L
$$

The $E_{x, y}^{d}\left(x, y, z, \omega_{1}\right)$ in the formula can be replaced by background field $E_{\mathrm{b}}\left(z_{0}\right)$.

$$
\begin{gathered}
\mathrm{E}_{\mathrm{b}}=\mathrm{E}_{\mathrm{b}}\left(Z_{0}\right) \exp \left(-k_{b}\right) \Delta z \\
\overline{\beta_{L}}(x, y, z)=\frac{1}{L}\left[\sum_{L=1}^{L} \beta_{L}(x, z, \omega)\right] \\
\beta_{\mathrm{s} b}=\left\{\frac{1+\bar{\beta}(\mathrm{x}, y, z)}{1-\bar{\beta}(\mathrm{x}, y, z)}\right\}^{2} \cdot \rho_{b}
\end{gathered}
$$

where $\rho_{\mathrm{b}}=1 / \sigma_{b}$.

\section{B. Digital Filter Spectrum Calculation}

According to the migration field calculation formula in frequency domain:

$$
E^{\mathrm{m}}\left(x_{\lambda}^{\prime}, y_{\lambda}^{\prime}\right)=\sum_{-\infty-\infty}^{\infty} \sum_{-\infty}^{\infty} C_{\mathrm{n} 1} E^{*}\left(x_{\lambda}^{\prime}-n \Delta x_{\lambda}, y_{\lambda}^{\prime}-l \Delta y_{\lambda}, z_{\lambda b}\right)
$$

Using Fourier transform, migration filter spectral value in the spatial frequency domain is as follows:

$$
S_{\mathrm{m}}\left(k_{\lambda x}, k_{\lambda y}, k_{b}, z_{\lambda}\right)=\exp \left[-v_{b}\left(z-z_{b}\right)\right]
$$

\section{Digital Filter Parameter Analysis}

Before applying the filter, the analysis of numerical filter parameter is needed. Its purpose is:

(1) Compare spectral characteristics of migration transform digital filter and analytical filter

(2) Analyze if the digital filters is resonable

(3) Discuss how to choose the filter parameters in the process of migration transform

The digital filter spectral analysis show that sampling distance cannot be larger than migration distance, and the size of the filter window should be increased when we using lowfrequency signal.

\section{Simple Three-dimensional Geoelectric Model Migration Imaging}

We use the above migration method to process the numeric data of geoelectric model in the whole space with the transmiter-receiver distance vary from near field area to thousands of kilometers and the operating frequency range is $0.1 \mathrm{~Hz}-10 \mathrm{~Hz}$. The geoelectric model used in this article and the migration results are shown below:

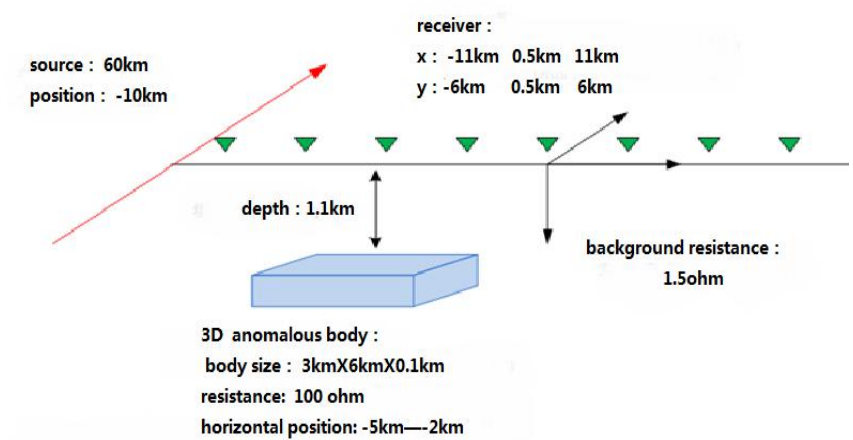

frequency range : $0.01 \mathrm{~Hz}-10 \mathrm{~Hz}$

frequencies : $0.010 .01770 .03160 .0562 \quad 0.10 .17780 .31620 .56321 .01 .7783 \quad 3.1623 \quad 5.623410$

Fig. 1. Low resistance surrounding rock model diagram 

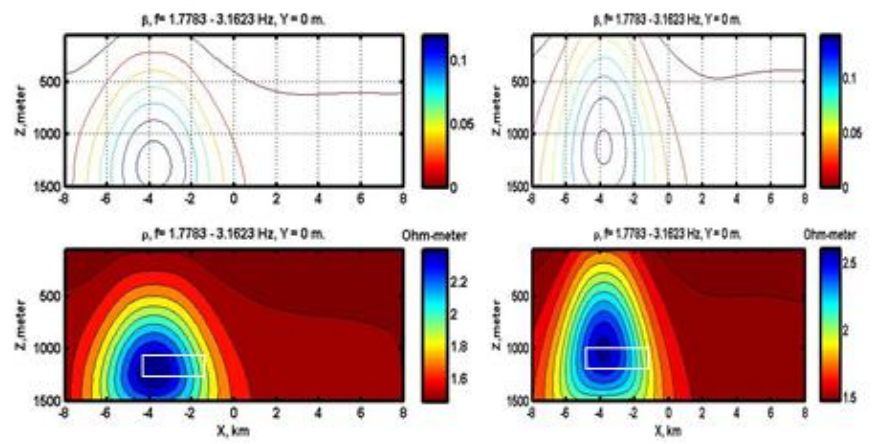

Dual Frequency migration

Dual Frequency migration

Fig. 2. The results of three-dimensional migration imaging

\section{E. Complex Three-dimensional Geoelectric Model Migration Imaging}

Low resistance surrounding rock with high resistance complex trapezoid model is as follows:

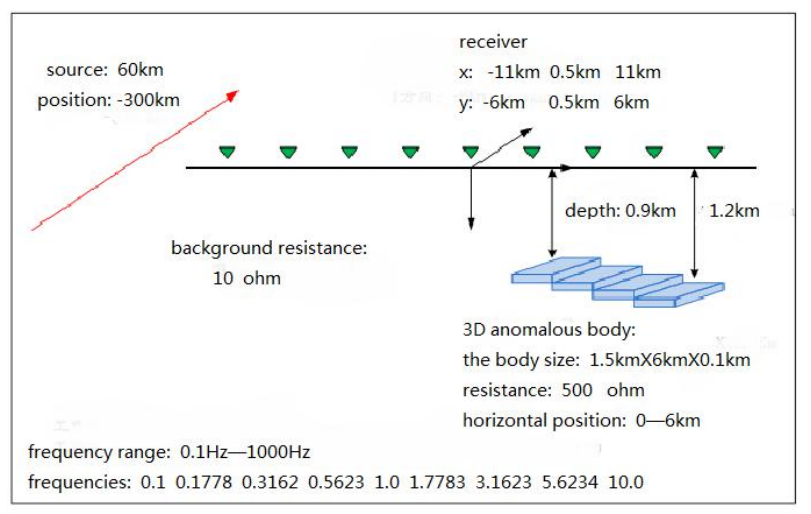

Fig. 3. Complex trapezoid model
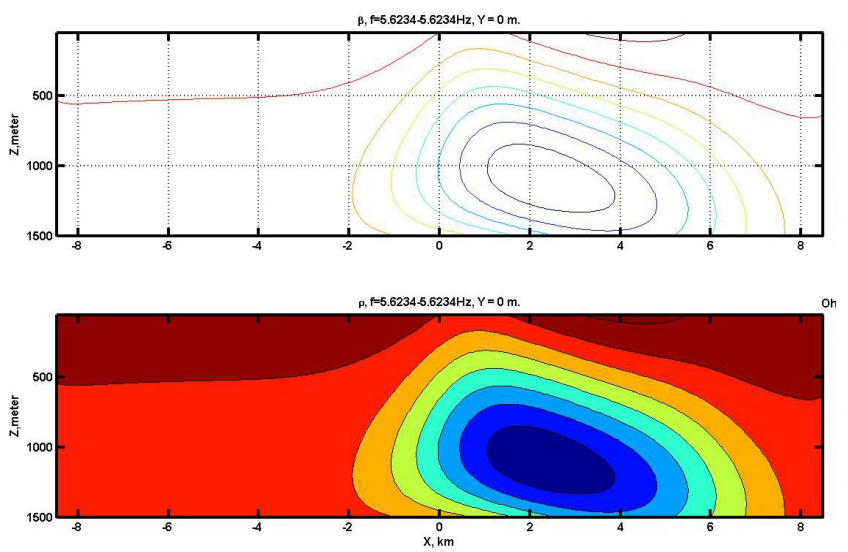

Fig. 4. Migration imaging of trapezoid model simulation data

From the above figure, one can see that the migration imaging technique can get the accurate position and basic shape of subsurface exploration body. The numerical experiments prove that migration technology a reliable and stable electromagnetic data interpretation method.

\section{Directive Time Domain Migration Field CALCUlation InTEGRAL TRANSFORMATION}

The method of solving the migration field based on Green's functions are called Integral transformation which was put forward by the Zhdanov et al in 2007 . When considering a two-dimensional form of the adjoint equation of Green's function, it satisfies the following equation :

$$
\nabla^{2} G^{2 \mathrm{~d}}+\mu_{0} \sigma_{0} \frac{\partial G^{2 \mathrm{~d}}}{\partial t}=\delta\left(\overrightarrow{\mathrm{r}}-\overrightarrow{r^{\prime}}\right) \delta\left(t-\mathrm{t}^{\prime}\right)
$$

A clear formula to directly calculate the two-dimensional electromagnetic migration field can be obtained. The final migration field solution of the problem has the form:

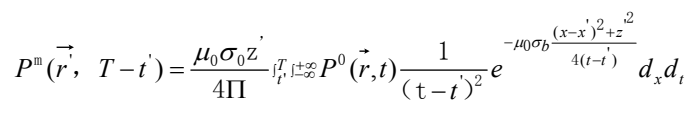

From above equation, the migration electromagnetic field can be understood as a special form of integral transform of the surface observation data, which is why the method of solving migration field is called the integral transform method.

\section{A. Integral Kernel Function Features}

The calculation of the electromagnetic migration fields is to process the surface observation data by using a special integral transformation, wherein the kernel function $\mathrm{Km}$ (Migration Kernel) of integral transform is expressed in the form below:

$$
K_{m}=\frac{1}{\left(t-t^{\prime}\right)^{2}} e^{-\mu_{0} \sigma_{b} \frac{\left(x^{\prime}-x\right)^{2}+z^{\prime 2}}{4\left(t-t^{\prime}\right)}}
$$

\section{B. Geoelectric Model}

The model shown here is a high resistance plate-like body, located in a uniform half-space with low resistivity surrounding rock. Schematic model is shown in Fig.5.

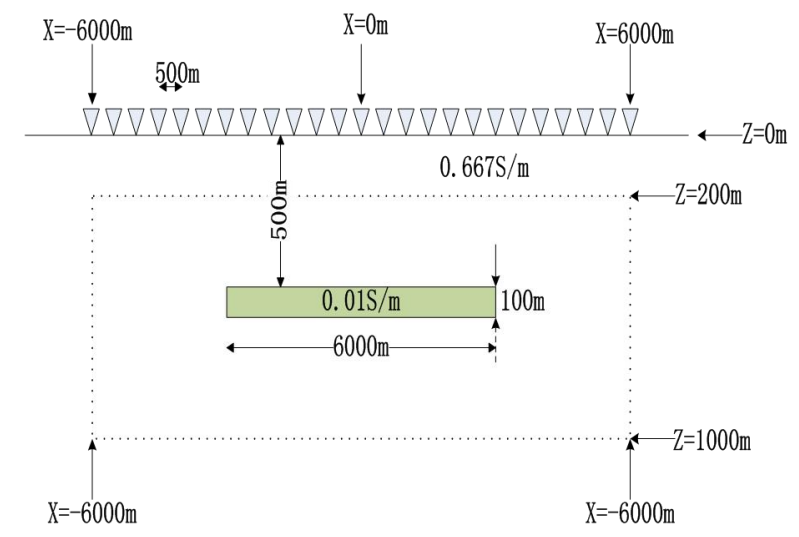

Fig. 5. Schematic diagram of time domain electromagnetic theory mode 
Selecting the background conductivity equal to half of the actual background conductivity, we calculated the migration field distribution for different migration time in subsurface media, which shows migration field changes with time. Finally we get the following stable migration field distribution.

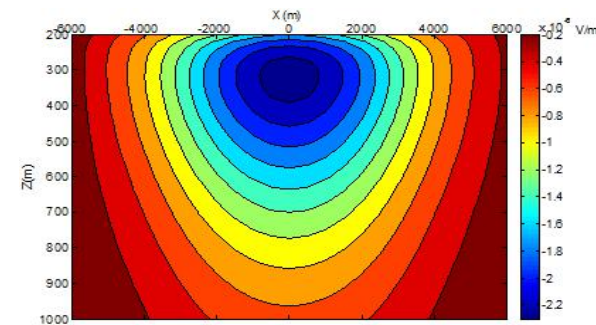

Fig. 6. migration field distribution

As can be seen from Fig.6, the migration field reasonably outlined the distribution of high resistance abnormal body in low resistance surrounding rock, and the migration calculation is stable.

\section{CONCLUSION}

Migration imaging of electromagnetic data is in time domain is a kind of stable and efficient way to explain electromagnetic data. This study showed that both time domain migration methods, filtering migration based on Laplace transform and the direct time domain method based on integral equation, can effectively achieve the position and basic shape of underground anomalies.

\section{REFERENCES}

[1] Michael S. Zhdanov, Peter N. Traynin, Oleg Portniaguine, Resistivity imaging by Time Domain Electromagnetic Migration(TDEMM). Exploration Geophysics, 1995,26, 186 194.

[2] Michael S. Zhdanov, Oleg Portniaguine, Time-domain electromagnetic migration in the solution of inverse problems. Geophys. J. Int. 1997,131, 293 309

[3] Newman G A, Commer M. New advances in three dimensional transient electromagnetic inversion[J]. Geophysical Journal International. 2005, 160(1): 5-32.

[4] Barnett C T. Simple inversion of time-domain electromagnetic data[J]. Geophysics. 1984, 49(7): 925-933

[5] Velikhov Y P, Zhdanov M S, Frenkel M A. Interpretation of MHDsounding data from the Kola Peninsula by the electromagnetic migration method[J]. Physics of the earth and planetary interiors. 1987, 45(2): 149160.

[6] Zhdanov M S, Booker J R. Underground Imaging By Electromagnetic Migration[C]. 1993.

[7] Zhdanov M S, Traynin P, Portniaguine O, et al. Time domain electromagnetic migration in INEL RWMC Cold Test Pit characterization[C]. 1995.

[8] Zhdanov M S, Traynin P. Inversion versus migration in two-dimensional geoelectrical problems[C]. Society of Exploration Geophysicists, 1996.

[9] Zhdanov M S, Li W D. 2D finite-difference time domain electromagnetic migration[J]. 67th SEG EM2.1. 1997: 370-373.

[10] Zhdanov M S, Li W S, Shiga N S. Preconditioned time domain electromagnetic migration[C]. 1998.

[11] Zhdanov M S, Pavlov D, Ellis R. Fast imaging of TDEM data by 2.5-D finite difference electromagnetic migration[C]. Society of Exploration Geophysicists, 2001. 